\title{
Decision Procedures for Inductive Boolean Functions Based on Alternating Automata
}

\author{
Abdelwaheb Ayari, David Basin, and Felix Klaedtke \\ Institut für Informatik, Albert-Ludwigs-Universität Freiburg, Germany.
}

\begin{abstract}
We show how alternating automata provide decision procedures for the equivalence of inductively defined Boolean functions that are useful for reasoning about parameterized families of circuits. We use alternating word automata to formalize families of linearly structured circuits and alternating tree automata to formalize families of tree structured circuits. We provide complexity bounds and show how our decision procedures can be implemented using BDDs. In comparison to previous work, our approach is simpler, yields better complexity bounds, and, in the case of tree structured families, is more general.
\end{abstract}

\section{Introduction}

Reasoning about parametric system descriptions is important in building scalable systems and generic designs. In hardware verification, the problem arises in verification of parametric combinational circuit families, for example, proving that circuits in one family are equivalent to circuits in another, for every parameter value. Another application of parametric reasoning is in establishing properties of sequential circuits, where time is the parameter considered. In this paper we present a new approach to these problems based on alternating automata on words and trees.

The starting point for our research is the work of Gupta and Fisher [6,7]. They developed a formalism for describing circuit families using one of two kinds of inductively defined Boolean functions. The first, called Linearly Inductive Boolean Functions, or LIFs, formalizes families of linearly structured circuits. The second, called Exponentially Inductive Boolean Functions, or EIFs, models families of tree structured circuits. As simple examples, consider the linear (serial) and tree structured 4-bit parity circuits described by the following diagrams.
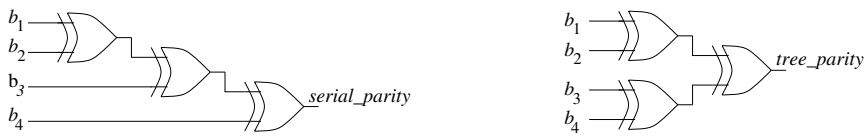

A LIF describing the general case of the linear circuit is given by the following equations. (We will formally introduce slightly different syntax in 93 and \$4)

$$
\begin{aligned}
\text { serial_parity }^{1}\left(b_{1}\right) & =b_{1} \\
\text { serial_parity }^{n}\left(b_{1}, \ldots, b_{n}\right) & =b_{n} \oplus \text { serial_parity }^{n-1}\left(b_{1}, \ldots, b_{n-1}\right) \quad \text { for } n>1 .
\end{aligned}
$$


Similarly, an EIF describing the family of tree-structured parity circuits is:

$$
\begin{aligned}
\text { tree_parity }^{1}\left(b_{1}\right)= & b_{1} \\
\text { tree_parity }^{2^{n}}\left(b_{1}, \ldots, b_{2^{n}}\right)= & \text { tree_parity }^{2^{n-1}}\left(b_{1}, \ldots, b_{2^{n-1}}\right) \oplus \\
& \text { tree_parity }^{2^{n-1}}\left(b_{2^{n-1}+1}, \ldots, b_{2^{n}}\right) \quad \text { for } n \geq 1 .
\end{aligned}
$$

Gupta and Fisher developed algorithms to translate these descriptions into novel data-structures that generalize BDDs (roughly speaking, their data-structures have additional pointers between BDDs, which formalize recursion). The resulting data-structures are canonical: different descriptions of the same family are converted into identical data-structures. This yields a decision procedure both for the equivalence of LIFs and for EIFs.

Motivated by their results, we take a different approach. We show how LIFs and EIFs can be translated, respectively, into alternating word and tree automata, whereby the decision problems for LIFs and EIFs are solvable by automata calculations. For LIFs, the translation and decision procedure are quite direct and may be implemented and analyzed using standard algorithms and results for word automata. For EIFs, the situation is more subtle since input is given by trees where only leaves are labeled by data and we are only interested in the equality of complete trees. Here, we decide equality using a procedure that determines whether a tree automaton accepts a complete leaf-labeled tree.

The use of alternating automata has a number of advantages. First, it gives us a simple view of (and leads to simpler formalisms for) LIFs and EIFs based on standard results from automata theory. For example, the expressiveness of these languages trivially falls out of our translations: LIFs describe regular languages on words and EIFs describe regular languages on trees (modulo the subtleties alluded to above). Hence, LIFs and EIFs can formalize any circuit family whose behavior is regular in the language theoretic sense. Second, it provides a handle on the complexity of the problems. For LIFs we show that the equality problem is PSPACE-complete and for EIFs it is in EXPSPACE. The result for LIFs represents a doubly exponential improvement over the previous results of Gupta and Fisher and our results for EIFs, are to our knowledge, the first published bounds for this problem. Finally, the use of alternating automata provides a basis for adapting data-structures recently developed in the MoNA project [10]; in their work, as well as ours, BDDs are used to represent automata and can often exponentially compress the representation of the transition function. We show that the use of BDDs to represent alternating automata offers similar advantages and plays an important rôle in the practical use of these techniques.

We proceed as follows. In $₫ 2$ we provide background material on word and tree automata. In $\$ 3$ and $\$$ we formalize LIFs and EIFs and explain our decision procedures. In \$5 we make comparisons and in \$6 draw conclusions and discuss future work. 


\section{Background}

Boolean Logic The set $B(V)$ of Boolean formulae (over $V$ ) is built from the constants 0 and 1 , variables $v \in V$, and the connectives $\neg, \vee, \wedge, \leftrightarrow$ and $\oplus$. For $\beta \in B(V), \beta\left[\alpha_{1} / v_{1}, \ldots, \alpha_{n} / v_{n}\right]$ denotes the formula where the $v_{i} \in V$ are simultaneously replaced by the formulae $\alpha_{i} \in B(V)$.

Boolean formulae are interpreted in the set $\mathbb{B}=\{0,1\}$ of truth values. A substitution is a function $\sigma: V \rightarrow \mathbb{B}$ that is homomorphically extended to $B(V)$. For $\sigma: V \rightarrow \mathbb{B}$ and $\beta \in B(V)$ we write $\sigma=\beta$ if $\sigma(\beta)=1$. We will sometimes identify a subset $M$ of $V$ with the substitution $\sigma_{M}: V \rightarrow \mathbb{B}$, where $\sigma_{M}(v)=1$ iff $v \in M$. For example, for the formula $v_{1} \oplus v_{2}$, we have $\left\{v_{1}\right\} \models v_{1} \oplus v_{2}$ but $\left\{v_{1}, v_{2}\right\} \not \forall v_{1} \oplus v_{2}$.

Words and Trees $\Sigma^{*}$ is the set of all words over the alphabet $\Sigma$. We write $\lambda$ for the empty word and $\Sigma^{+}$for $\Sigma^{*} \backslash\{\lambda\}$. For $u, v \in \Sigma^{*}$, u.v denotes concatenation, $|u|$ denotes $u$ 's length, and $u^{\mathrm{R}}$ denotes the reversal of $u$.

A $\Sigma$-labeled tree (with branching factor $r \in \mathbb{N}$ ) is a function $t$ where the range of $t$ is $\Sigma$ and the domain of $t, \operatorname{dom}(t)$ for short, is a finite subset of $\{0, \ldots, r-1\}^{*}$ where (i) $\operatorname{dom}(t)$ is prefix closed and (ii) if $u . i \in \operatorname{dom}(t)$, then $u . j \in \operatorname{dom}(t)$ for all $j<i$. The elements of $\operatorname{dom}(t)$ are called nodes and $\lambda \in \operatorname{dom}(t)$ is called the root. The node $u . i \in \operatorname{dom}(t)$ is a successor of $u$. A node is an inner node if it has successors and is a leaf otherwise. The height of $t$ is $|t|=\max (\{0\} \cup\{|u|+1 \mid u \in$ $\operatorname{dom}(t)\})$. The depth of a node $u \in \operatorname{dom}(t)$ is the length of $u$.

A tree is complete if all its leaves have the same depth. The frontier of $t$ is the word front $(t) \in \Sigma^{*}$ where the $i$ th letter is the label of the $i$ th leaf in $t$ (from the left). $\Sigma^{\mathrm{T} *}$ denotes the set of all binary $\Sigma$-labeled trees and $\Sigma^{\mathrm{T}+}$ is $\Sigma^{\mathrm{T} *}$ without the empty tree.

Nondeterministic Automata A nondeterministic word automaton (NWA) $\mathcal{A}$ is a tuple $\left(\Sigma, Q, q_{0}, F, \delta\right)$, where $\Sigma$ is a nonempty finite alphabet, $Q$ is a nonempty finite set of states, $q_{0} \in Q$ is the initial state, $F \subseteq Q$ is a set of accepting states, and $\delta: Q \times \Sigma \rightarrow \mathcal{P}(Q)$ is a transition function. A run of $\mathcal{A}$ on a word $w=a_{1} \ldots a_{n} \in \Sigma^{*}$ is a word $\pi=s_{1} \ldots s_{n+1} \in Q^{+}$with $s_{1}=q_{0}$ and $s_{i+1} \in \delta\left(s_{i}, a_{i}\right)$ for $1 \leq i \leq n$. $\pi$ is accepting if $s_{n+1} \in F$. A word $w$ is accepted by $\mathcal{A}$ if there is an accepting run of $\mathcal{A}$ on $w ; L(\mathcal{A})$ denotes the set of accepted words.

Nondeterministic (top-down, binary) tree automata (NTA) are defined analogously: $\mathcal{A}$ is a tuple $\left(\Sigma, Q, q_{0}, F, \delta\right)$, where $\Sigma, Q, q_{0}$ and $F$ are as before. The transition function is $\delta: Q \times \Sigma \rightarrow \mathcal{P}(Q \times Q)$. A run of a NTA $\mathcal{A}$ on a tree $t \in \Sigma^{\mathrm{T} *}$ is a tree $\pi \in Q^{\mathrm{T}+}$, where $\operatorname{dom}(\pi)=\{\lambda\} \cup\{w \cdot b \mid w \in \operatorname{dom}(t)$ and $b \in\{0,1\}$. Moreover, $\pi(\lambda)=q_{0}$ and for $w \in \operatorname{dom}(t),(\pi(w .0), \pi(w .1)) \in \delta(\pi(w), t(w))$. The run $\pi$ is accepting if $\pi(w) \in F$ for any leaf $w \in \operatorname{dom}(\pi)$. A tree $t$ is accepted by $\mathcal{A}$ if there is an accepting run of $\mathcal{A}$ on $t ; L(\mathcal{A})$ denotes the set of accepted trees. 
NWAs and NTAs recognize the regular word and tree languages and are effectively closed under intersection, union, complement and projection. For a detailed account of regular word and tree languages see [1] and 4] respectively.

Alternating Automata Alternating automata for words were introduced in [2, 3] and for trees in [15. We use the definition of alternating automata for words from [17] and generalize it to trees. For this we need the notion of the positive Boolean formulae: Let $B^{+}(V)$ be the set of Boolean formulae built from 0,1 , $v \in V$, and the connectives $\vee$ and $\wedge$.

An alternating word automaton $(A W A)$ is of the form $\mathcal{A}=\left(\Sigma, Q, q_{0}, F, \delta\right)$ where everything is as before, except for the transition function $\delta: Q \times \Sigma \rightarrow$ $B^{+}(Q)$. The same holds for alternating tree automata (ATA) where the only difference is the transition function $\delta: Q \times \Sigma \rightarrow B^{+}(Q \times\{L, R\})$. We write $q^{X}$ for $(q, X) \in Q \times\{L, R\}$.

We will only define a run for an ATA; the restriction to AWA is straightforward. For an ATA, a run $\pi$ of $\mathcal{A}$ on $t \in \Sigma^{\mathrm{T} *}$ is a $Q \times\{0,1\}^{*}$-labeled tree, with $\pi(\lambda)=\left(q_{0}, \lambda\right)$. Moreover, for each node $w \in \operatorname{dom}(\pi)$, with $\pi(w)=(q, u)$, and for all of the $r \in \mathbb{N}$ successor nodes of $w$ :

$$
\begin{aligned}
& \left\{p^{L} \mid \pi(w . k)=(p, u .0) \text { for } 0 \leq k<r\right\} \cup \\
& \left\{p^{R} \mid \pi(w . k)=(p, u .1) \text { for } 0 \leq k<r\right\} \models \delta(q, t(u)) .
\end{aligned}
$$

$\pi$ is accepting if for every leaf $w$ in $\pi$, where $\pi(w)=(p, u . k), u$ is leaf in $t$ and $p \in F$. The tree language accepted by $\mathcal{A}$ is $L(\mathcal{A})=\left\{t \in \Sigma^{\mathrm{T} *} \mid \mathcal{A}\right.$ accepts $\left.t\right\}$. If there exists an accepting run of $\mathcal{A}^{\prime}=(\Sigma, Q, q, F, \delta)$ for $q \in Q$ on $t$, then we say that $\mathcal{A}$ accepts $t$ from $q$. We use the same terminology for AWAs.

It is straightforward to construct an alternating automaton from a nondeterministic automaton of the same size. Conversely, given an AWA one can construct an equivalent NWA with at most exponentially more states [2 317]. The states of the nondeterministic automaton are the interpretations of the Boolean formulae of the alternating automaton's transition function. This construction can be generalized to tree automata. Hence alternation does not increase the expressiveness of word and tree automata but, as we will see, it does enhance their ability to model problems.

\section{Linearly Inductive Boolean Functions}

\subsection{Definition of LIFs}

We now define linearly inductive Boolean functions. Our definition differs slightly from [6 7 8], however they are equivalent (see \$5]).

Syntax Let the two sets $V=\left\{v_{1}, \ldots, v_{r}\right\}$ and $F=\left\{f_{1}, \ldots, f_{s}\right\}$ be fixed for the remainder of this paper. 
A LIF formula (over $V$ and $F$ ) is a pair $(\alpha, \beta)$, with $\alpha \in B(V)$ and $\beta \in$ $B(V \uplus F)$. The formulae $\alpha$ and $\beta$ formalize the base and step case of a recursive definition. A LIF system (over $V$ and $F$ ) is a pair $(\mathrm{F}, \eta)$ where $\mathrm{F}$ is a set of LIF formulae over $V$ and $F$ and $\eta: F \rightarrow F$. That is, $\eta$ assigns each $f \in F$ a LIF formula $(\alpha, \beta) \in \mathrm{F}$. We will write $\left(\alpha_{f}, \beta_{f}\right)$ for $\eta(f)=(\alpha, \beta)$ and omit $V$ and $F$ when they are clear from the context.

Semantics Let $\mathcal{S}$ be a LIF system. An evaluation of $\mathcal{S}$ on $w=b_{1} \ldots b_{n} \in\left(\mathbb{B}^{r}\right)^{+}$ is a word $d_{1} \ldots d_{n} \in\left(\mathbb{B}^{s}\right)^{+}$such that for $b_{i}=\left(a_{1}^{i}, \ldots, a_{r}^{i}\right), d_{i}=\left(c_{1}^{i}, \ldots, c_{s}^{i}\right)$, and $1 \leq k \leq s$ :

$$
c_{k}^{1}=1 \quad \text { iff } \quad\left\{v_{l} \mid a_{l}^{1}=1, \text { for } 1 \leq l \leq r\right\} \models \alpha_{f_{k}},
$$

and for all $i, 1<i \leq n$,

$$
\begin{aligned}
c_{k}^{i}=1 \quad \text { iff } \quad & \left\{v_{l} \mid a_{l}^{i}=1, \text { for } 1 \leq l \leq r\right\} \cup \\
& \left\{f_{l} \mid c_{l}^{i-1}=1, \text { for } 1 \leq l \leq s\right\} \models \beta_{f_{k}} .
\end{aligned}
$$

An easy induction over the length of $w$ shows:

Lemma 1. For $\mathcal{S}$ a LIF system and $w \in\left(\mathbb{B}^{r}\right)^{+}$, the evaluation of $\mathcal{S}$ on $w$ is uniquely defined.

Hence $f_{k} \in F$ together with $\mathcal{S}$ determine a function $f_{k}^{\mathcal{S}}:\left(\mathbb{B}^{r}\right)^{+} \rightarrow \mathbb{B}$. Namely, for $w \in\left(\mathbb{B}^{r}\right)^{+}, f_{k}^{\mathcal{S}}(w)=c_{k}^{|w|}$. We call $f_{k}^{\mathcal{S}}$ the $L I F$ of $\mathcal{S}$ and $f_{k}$. When $\mathcal{S}$ is clear from the context, we omit it.

Examples We present three simple examples. First, for $V=\{x\}$ and $F=$ $\{$ serial_parity $\}$, the following LIF system $\mathcal{S}_{1}$ formalizes the family of linear parity circuits given in the introduction.

$$
\alpha_{\text {serial_parity }}=x \quad \beta_{\text {serial_parity }}=x \oplus \text { serial_parity }
$$

In particular, serial_parity $\mathcal{S}^{\mathcal{S}_{1}}$ applied to $b_{1} \ldots b_{n} \in \mathbb{B}^{+}$equals parity $^{n}\left(b_{1}, \ldots, b_{n}\right)$.

The second LIF system $\mathcal{S}_{2}$ over $V=\{a, b, c i n\}$ and $F=\{$ sum, carry $\}$ formalizes a family of ripple-carry adders.

$$
\begin{array}{ll}
\alpha_{\text {sum }}=(a \oplus b) \oplus \text { cin } & \beta_{\text {sum }}=(a \oplus b) \oplus \text { carry } \\
\alpha_{\text {carry }}=(a \wedge b) \vee((a \vee b) \wedge \text { cin }) & \beta_{\text {carry }}=(a \wedge b) \vee((a \vee b) \wedge \text { carry })
\end{array}
$$

Here $\operatorname{sum}^{\mathcal{S}_{2}}$ [respectively carry $^{\mathcal{S}_{2}}$ ] represents the adder's $n$th output bit [respectively carry bit].

The third example shows how to describe a sequential circuit by a LIF system. The LIF system $\mathcal{S}_{3}$ over $V=\{e\}$ and $F=\left\{Y_{1}, Y_{2}, Y_{3}\right\}$ describes a 3-bit counter with an enable bit.

$$
\begin{array}{ll}
\alpha_{Y_{1}}=0 & \beta_{Y_{1}}=\left(e \wedge \neg Y_{1}\right) \vee\left(\neg e \wedge Y_{1}\right) \\
\alpha_{Y_{2}}=0 & \beta_{Y_{2}}=\left(e \wedge\left(Y_{1} \oplus Y_{2}\right)\right) \vee\left(\neg e \wedge Y_{2}\right) \\
\alpha_{Y_{3}}=0 & \beta_{Y_{3}}=\left(e \wedge\left(\left(Y_{1} \wedge Y_{2}\right) \oplus Y_{3}\right)\right) \vee\left(\neg e \wedge Y_{3}\right) .
\end{array}
$$


$Y_{i}^{\mathcal{S}_{3}}(w)$, with $w \in \mathbb{B}^{+}$, is the value of the $i$ th output bit at time $|w|$ of the 3-bit counter, where $w$ encodes the enable input signals.

\subsection{Equivalence of LIF Systems and AWAs}

A function $g:\left(\mathbb{B}^{r}\right)^{+} \rightarrow \mathbb{B}$ is LIF-representable if there exists a LIF system $\mathcal{S}$ and a $f \in F$, where $g(w)=f^{\mathcal{S}}(w)$ for all $w \in\left(\mathbb{B}^{r}\right)^{+}$. A language $L \subseteq\left(\mathbb{B}^{r}\right)^{+}$ is LIF-representable if its characteristic function, $g(w)=1$ iff $w \in L$, is LIFrepresentable. Gupta and Fisher have shown in 69. that any LIF-representable language is regular. They prove that their data-structure for representing a LIF system corresponds to a minimal deterministic automaton that accepts the language $\left\{w^{\mathrm{R}} \mid f^{\mathcal{S}}(w)=1\right.$, for $\left.w \in\left(\mathbb{B}^{r}\right)^{+}\right\}$.

We present here a simpler proof of regularity by showing that LIF systems directly correspond to AWAs. We also prove a weakened form of the converse: almost all regular languages are LIF-representable. The weakening though is trivial and concerns the empty word, and if we consider languages without the empty word we have an equivalence 1 Hence, for the remainder of this secction, we consider only automata (languages) that do not accept (include) the empty word $\lambda$.

For technical reasons we will work with LIF systems in a kind of negation normal form. A Boolean formula $\beta \in B(X)$ is positive in $Y \subseteq X$ if negations occur only directly in front of the Boolean variables $v \in X \backslash Y$ and, furthermore, the only connectives allowed are $\neg, \wedge$ and $\vee$. A LIF system $\mathcal{S}$ is in normal form if $\beta_{f}$ is positive in $F$, for each $f \in F$.

Lemma 2. Let $\mathcal{S}$ be a LIF system over $V$ and $F$. Then there is a LIF system $\mathcal{S}^{\prime}$ over $V$ and $F^{\prime}=F \uplus\{\bar{f} \mid f \in F\}$ in normal form where, for all $f \in F$ and $w \in\left(\mathbb{B}^{r}\right)^{+}, f^{\mathcal{S}^{\prime}}(w)=f^{\mathcal{S}}(w)$ and $\bar{f}^{\mathcal{S}^{\prime}}(w)=1$ iff $f^{\mathcal{S}}(w)=0$

Proof. Without loss of generality, we assume that for $\beta \in B(X)$ only the connectives $\neg, \vee$, and $\wedge$ occur. The other connectives can be eliminated as standard, which may lead to exponentially larger formulae. By $\operatorname{nnf}(\beta)$ we denote the negation normal form of $\beta \in B(X)$.

By using the same idea as [3], it is easy to construct a LIF system $\mathcal{S}^{\prime}$ by introducing for each $f \in F$ a new variable $\bar{f}$ that "simulates" $\neg f$. Let $\mathcal{S}=(\mathrm{F}, \eta)$. For $f \in F$, with $\eta(f)=(\alpha, \beta)$, the mapping $\eta^{\prime}$ of the LIF system $\mathcal{S}^{\prime}$ is defined by $\eta^{\prime}(f)=(\alpha, \gamma)$ and $\eta^{\prime}(\bar{f})=(\neg \alpha, \bar{\gamma})$ where $\gamma$ and $\bar{\gamma}$ are obtained from $\operatorname{nnf}(\beta)$, respectively $\operatorname{nnf}(\neg \beta)$, by replacing the sub-formulae $\neg f_{i}$ by $\bar{f}_{i}$.

We now prove that LIF-representable languages and ( $\lambda$-free) regular languages coincide.

\footnotetext{
${ }^{1}$ We can easily redefine LIFs to define functions over $\left(\mathbb{B}^{r}\right)^{*}$. However, following Gupta and Fisher we avoid this as the degenerate base case (0 length input) is ill-suited for modeling parametric circuits. Ignoring the empty word is immaterial for our complexity and algorithmic analysis.
} 
Theorem 1. LIF systems are equivalent to AWAs. In particular:

i) Given an $A W A \mathcal{A}=\left(\mathbb{B}^{r}, Q, q_{0}, F, \delta\right)$, there is a LIF system $\mathcal{S}$ over $V=$ $\left\{v_{1}, \ldots, v_{r}\right\}$ and $Q$ in normal form such that for all $w \in\left(\mathbb{B}^{r}\right)^{+}$and $q \in Q$,

$$
q^{\mathcal{S}}(w)=1 \quad \text { iff } \quad \mathcal{A} \text { accepts } w^{\mathrm{R}} \text { from } q .
$$

ii) Given a LIF system $\mathcal{S}$ in normal form over $V$ and $F$, there exists an $A W A$ $\mathcal{A}$ with states $F \uplus\left\{q_{\text {base }}, q_{\text {step }}\right\}$ such that for all $w \in\left(\mathbb{B}^{r}\right)^{+}$and $f \in F$,

$$
\mathcal{A} \text { accepts } w \text { from } f \quad \text { iff } \quad f^{\mathcal{S}}\left(w^{\mathrm{R}}\right)=1 \text {. }
$$

Proof. (i) We encode each $b \in \mathbb{B}^{r}$ by a formula $\gamma_{b} \in B(V)$. For example, $(0,1,1,0) \in \mathbb{B}^{4}$ is encoded as the Boolean formula $\gamma_{(0,1,1,0)}=\neg v_{1} \wedge v_{2} \wedge v_{3} \wedge \neg v_{4}$. The LIF formula for $q$ in $\mathcal{S}$ is

$$
\alpha_{q}=\bigvee_{b \in \mathbb{B}^{r}}\left(\gamma_{b} \wedge B(q, b)\right) \quad \beta_{q}=\bigvee_{b \in \mathbb{B}^{r}}\left(\gamma_{b} \wedge \delta(q, b)\right)
$$

with $B(q, b)=1$ iff $F \models \delta(q, b)$. Here, the Boolean formula $\beta_{q}$ simulates the transition from the state $q$ on a non-final letter of the input word. The final state set $F$ is simulated by the Boolean formula $\alpha_{q}$, i.e., $F \models \delta(q, b)$ iff $\left\{v_{i} \mid b_{i}=\right.$ $1\} \models \alpha_{q}$.

We prove (i) by induction over the length of $w \in\left(\mathbb{B}^{r}\right)^{+}$. If $|w|=1$, then the equivalence follows from the definition of $\alpha_{q}$, for any $q \in Q$. Assume (i) is true for the word $w$, i.e., for each $q_{k} \in Q, \mathcal{A}$ accepts $w^{\mathrm{R}}$ from $q_{k}$ iff $q_{k}^{\mathcal{S}}(w)=1$. Let $u . d$ be an evaluation of $\mathcal{S}$ on $w$ with $d=\left(c_{1}, \ldots, c_{|Q|}\right)$. It holds that $q_{k}^{\mathcal{S}}(w)=1$ iff $c_{k}=1$. We prove (i) for $w . b$ with $b=\left(a_{1}, \ldots, a_{r}\right)$. As defined, for each $q \in Q$ we have $q^{\mathcal{S}}(w . b)=1$ iff

$$
\left\{v_{l} \mid a_{l}=1, \text { for } 1 \leq l \leq r\right\} \cup\left\{q_{l} \mid c_{l}=1 \text {, for } 1 \leq l \leq|Q|\right\} \models \bigvee_{b^{\prime} \in \mathbb{B}^{r}}\left(\gamma_{b^{\prime}} \wedge \delta\left(q, b^{\prime}\right)\right)
$$

By the induction hypothesis, we obtain $\left\{q_{l} \mid \mathcal{A}\right.$ accepts $w^{\mathrm{R}}$ from $q_{l}$, for $1 \leq l \leq$ $|Q|\} \models \delta\left(q_{k}, b\right)$. From this we can easily construct an accepting run of $\mathcal{A}$ from $q$ on $(w . b)^{\mathrm{R}}$. The other direction holds by definition of an accepting run.

(ii) For an arbitrary $g \in F$, let $\mathcal{A}=\left(\mathbb{B}^{r}, F \uplus\left\{q_{\text {base }}, q_{\text {step }}\right\}, g,\left\{q_{\text {base }}\right\}, \delta\right)$ with $\delta\left(q_{\text {base }}, b\right)=0, \delta\left(q_{\text {step }}, b\right)=q_{\text {base }} \vee q_{\text {step }}$, and for $f \in F$

$\delta\left(f,\left(b_{1}, \ldots, b_{r}\right)\right)=\left(q_{\text {step }} \wedge \beta_{f}\left[b_{1} / v_{1}, \ldots, b_{r} / v_{r}\right]\right) \vee \begin{cases}q_{\text {base }} & \text { if }\left\{v_{i} \mid b_{i}=1\right\} \models \alpha_{f} \\ 0 & \text { otherwise }\end{cases}$

Intuitively when $\mathcal{A}$ is in state $f \in F$ and reads $\left(b_{1}, \ldots, b_{r}\right) \in \mathbb{B}^{r}$ it guesses if the base case is reached. When this is the case, the next state is $q_{\text {base }}$ iff $\left\{v_{i} \mid b_{i}=1\right\} \models \alpha_{f}$. Otherwise, if the base case is not reached, the AWA proceeds according to the step case given by the Boolean formula $\beta_{f}$ of the LIF system. The equivalence is proved in a similar way to (i). 
Note that if a LIF formula only uses the connectives $\neg, \wedge$ and $\vee$, then, following the proof of Lemma 2 a normal form can be obtained in polynomial time. Moreover, if $V$ is fixed the size, the AWA $\mathcal{A}$ of Theorem 1 (ii) can be constructed in polynomial time, since the size of the alphabet $\mathbb{B}^{r}$ is a constant. However, if we allow $V$ to vary, then the size of the AWA constructed can be exponentially larger than the size of the LIF system, i.e. $|V|+|F|+\sum_{f \in F}\left(\left|\alpha_{f}\right|+\right.$ $\left.\left|\beta_{f}\right|\right)$, since the input alphabet of $\mathcal{A}$ is of size $2^{|V|}$.

\subsection{Deciding LIF Equality}

Given LIF systems $\mathcal{S}$ over $V$ and $F$, and $\mathcal{T}$ over $V$ and $G$, and function symbols $f_{k} \in F$ and $g_{l} \in G$, the equality problem for LIFs is to decide whether $f_{k}^{\mathcal{S}}(w)=$ $g_{l}^{\mathcal{T}}(w)$, for all $w \in\left(\mathbb{B}^{r}\right)^{+}$. We first show that this problem is PSPACE-complete and afterwards show how, using BDDs, the construction in Theorem 1 provides the basis for an efficient implementation.

\section{Theorem 2. The equality problem for LIFs is PSPACE-complete.}

Proof. We reduce the emptiness problem for AWAs, which is PSPACE-hard [12, 17, to the equality problem for LIFs. Given an AWA $\mathcal{A}$ with initial state $q_{0}$, by Theorem [1(i) we can construct an equivalent LIF system $\mathcal{S}$ in polynomial time. Let the LIF system $\mathcal{T}$ be given by the formulae $\alpha_{g}=0$ and $\beta_{g}=0$. Then $q_{0}^{\mathcal{S}}=g^{\mathcal{T}}$ iff $L(\mathcal{A})=\emptyset$.

Theorem 1(ii) cannot be used to show that the problem is in PSPACE because, as explained in the previous section, both the normal form and the size of the two constructed AWAs can be exponentially larger than the size of the LIF instances. Hence, we instead give a direct proof. The following Turing machine $\mathcal{M}$ accepts a problem instance in PSPACE iff a word $w=b_{1} \ldots b_{n} \in\left(\mathbb{B}^{r}\right)^{+}$ exists with $f_{k}^{\mathcal{S}}(w) \neq g_{l}^{\mathcal{T}}(w)$. Let $d_{1} \ldots d_{n} \in\left(\mathbb{B}^{|F|}\right)^{+}$be the evaluation of $\mathcal{S}$ on $w$ and $d_{1}^{\prime} \ldots d_{n}^{\prime} \in\left(\mathbb{B}^{|G|}\right)^{+}$be the evaluation of $\mathcal{T}$ on $w$. $\mathcal{M}$ guesses in the $i$ th step $b_{i} \in \mathbb{B}^{r}$ and calculates $d_{i}=\left(c_{1}, \ldots, c_{|F|}\right)$ and $d_{i}^{\prime}=\left(c_{1}^{\prime}, \ldots, c_{|G|}^{\prime}\right)$ of the evaluations. If $c_{k} \neq c_{l}^{\prime}$ then $\mathcal{M}$ accepts the instance and otherwise $\mathcal{M}$ continues with the $(i+1)$ th step. Note that for the $i$ th step only $d_{i-1}$ and $d_{i-1}^{\prime}$ and $b_{i}$ are required to calculate $d_{i}$ and $d_{i}^{\prime}$. Hence $\mathcal{M}$ runs in polynomial space, since in the $i$ th step the space $|V|$ is required to store $b_{i}$ and the space $2(|F|+|G|)$ to store $d_{i-1}, d_{i}$, $d_{i-1}^{\prime}$, and $d_{i}^{\prime} . \mathcal{M}$ needs linear time in the size of the LIF formulae of $\mathcal{S}$ and $\mathcal{T}$ to calculate $d_{i}$ and $d_{i}^{\prime}$ from $b_{i}, d_{i-1}$ and $d_{i-1}^{\prime}$. Since PSPACE is closed under nondeterminism and complementation, the equality problem for LIFs is in PSPACE. $\square$

Although the machinery of alternating automata may appear a bit heavy, it leads to simple translations as there is a direct correspondence between function symbols in a LIF system and states in the corresponding AWA. This would not be possible using nondeterministic automata. Because the emptiness problem for NWAs is LOGSPACE-complete and the equality problem for LIFs is PSPACEcomplete, a translation of a LIF system to a nondeterministic automata must, in general, lead to an exponential blow-up in the state space. 
InPUT: AWA $\mathcal{A}=\left(\Sigma, Q, q_{0}, F, \delta\right)$

Output: returns true iff $L(\mathcal{A})=\emptyset$

Current $:=\left\{\left\{q_{0}\right\}\right\}$

Processed $:=\emptyset$;

while Current $\neq \emptyset$ do begin

if Current $\cap \mathcal{P}(F) \neq \emptyset$ then return false;

else begin

Processed $:=$ Processed $\cup$ Current; end;

Current $:=\left\{T^{\prime} \subseteq Q\left|T^{\prime}\right|=\bigwedge_{q \in T} \delta(q, a)\right.$ for $T \in$ Current, $\left.a \in \Sigma\right\} \backslash$ Processed;

end;

return true;

Fig. 1. Decision procedure for the emptiness problem for AWAs.

Implementation In the proof of Theorem 2 we did not use the mapping from LIFs to AWAs given by Theorem 1(ii) due to the possible exponential blowup when normalizing the LIF system, and the certain exponential blow-up in representing the AWA's alphabet. We describe here how these blow-ups can sometimes be avoided by using BDDs.

The reduction of LIF equality to the emptiness problem for AWAs is straightforward. From the LIF systems $\mathcal{S}$ over $V$ and $F$, and $\mathcal{T}$ over $V$ and $G$ we construct the LIF system $\widetilde{\mathcal{S}}$ over $V$ and $\{\widetilde{f}\} \uplus F \uplus G$ with the additional LIF formula $\alpha_{\tilde{f}}=\neg\left(\alpha_{f} \leftrightarrow \alpha_{g}\right)$ and $\beta_{\widetilde{f}}=\neg\left(\beta_{f} \leftrightarrow \beta_{g}\right)$. We then normalize $\widetilde{\mathcal{S}}$ and use Theorem प(ii) to construct the AWA $\mathcal{A}$ with the initial state $\widetilde{f}$. By construction, $L(\mathcal{A}) \neq \emptyset$ iff $\widetilde{f^{\mathcal{S}}}(w)=1$ for some $w \in\left(\mathbb{B}^{r}\right)^{+}$iff $f^{\mathcal{S}} \neq g^{\mathcal{T}}$.

To decide if an AWA $\mathcal{A}=\left(\Sigma, Q, q_{0}, F, \delta\right)$ accepts the empty language, we construct "on-the-fly" the equivalent NWA $\mathcal{B}=\left(\Sigma, \mathcal{P}(Q),\left\{q_{0}\right\}, \mathcal{P}(F), \delta^{\prime}\right)$ with

$$
\delta^{\prime}(T, a)=\left\{T^{\prime} \subseteq Q \mid T^{\prime} \models \bigwedge_{q \in T} \delta(q, a)\right\},
$$

and search for a path from the initial state $\left\{q_{0}\right\}$ of $\mathcal{B}$ to a final state. We do this with a parallel breadth-first search in the state space of $\mathcal{B}$ as described in Figure 1.

To analyze the complexity, observe that the while-loop is traversed maximally $2^{|Q|}$-times and the calculation in each iteration requires $O\left(2^{|Q|}|\Sigma|\right)$-time. Hence the worst-case running time is $O\left(2^{2|Q|}|\Sigma|\right)$. We need two vectors of the length $2^{|Q|}$ to represent the sets Current and Processed. Hence the required space is the maximum of $O\left(2^{|Q|}\right)$ and the size of the representation of the AWA $\mathcal{A}$.

It is possible to use BDDs in two places to sometimes achieve an exponential savings in space. First, the sets Current, Processed $\subseteq \mathcal{P}(Q)$ can be encoded as BDDs where a BDD represents the characteristic function of the set. Second, since the size of $\mathcal{A}$ 's alphabet $\left(\mathbb{B}^{r}\right)$ is exponential in $|V|$, we use the same idea that Gupta and Fisher employed for their representation of LIFs: we need not 


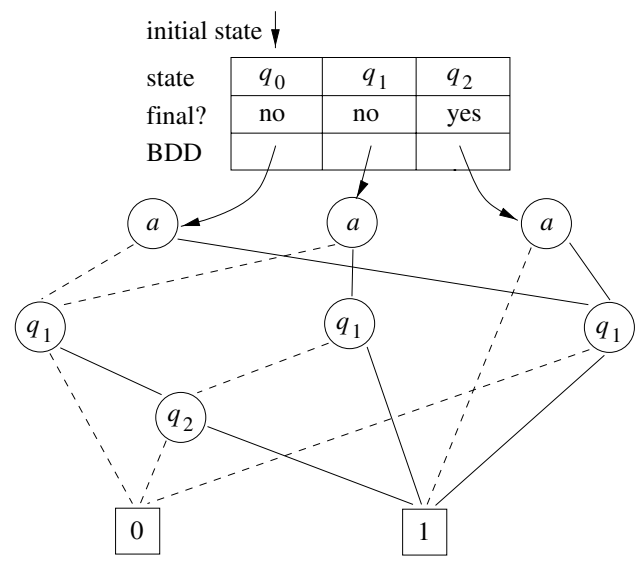

Fig. 2. Representation of an AWA.

explicitly represent the exponentially large alphabet and we can use BDDs to represent the transition function. For example, Figure 2 depicts the representation of the AWA $\mathcal{A}=\left(\mathbb{B},\left\{q_{0}, q_{1}, q_{2}\right\}, q_{0},\left\{q_{2}\right\}, \delta\right)$ with the transition function

$$
\begin{array}{llrl}
\delta\left(q_{0}, 0\right) & =q_{1} \wedge q_{2} & & \delta\left(q_{0}, 1\right)=q_{1} \\
\delta\left(q_{1}, 0\right) & =q_{1} \wedge q_{2} & & \delta\left(q_{1}, 1\right)=q_{1} \vee q_{2} \\
\delta\left(q_{2}, 0\right) & =1 & & \delta\left(q_{2}, 1\right)=q_{1} .
\end{array}
$$

The solid [respectively dashed] lines correspond to the variable assignment 1 [respectively 0]. For example, the state $q_{0}$ has a pointer to a BDD, where the first node (labeled $a$ ) encodes the alphabet; the solid line from this node points to a BDD representing $\delta\left(q_{0}, 1\right)=q_{1}$ and the dashed line points to a BDD representing $\delta\left(q_{0}, 0\right)=q_{1} \wedge q_{2}$.

We have implemented the emptiness test for AWAs using the CUDD package [16] and have begun preliminary testing and comparison. For the examples given in 3.1, building AWAs for the descriptions given and testing them for emptiness or equivalence with alternative descriptions is very fast: it takes a fraction of a second and most of the time is spent with $\mathrm{I} / \mathrm{O}$. We can carry out more ambitious tests by scaling up the sequential 3-bit counter example, namely performing tests on an $n$-bit counter for different values of $n$. This example is also interesting as it demonstrates the worst-case performance of our decision procedure since exponential many states of the NWA must be constructed to decide if the AWA describes the empty language.

Table 1 gives empirical results of the required space and time for the emptiness test for the resulting AWAs on a SUN Sparc Ultra with 250MHz. In the rightmost column are the running times on a SUN Sparc Ultra with $300 \mathrm{MHz}$ for building the canonical representation in Gupta and Fisher's approach. For large values of $n$, our approach yields significantly better results, although in both cases the algorithms require exponential time and space. Note that some 


\begin{tabular}{l|ccc|c}
$n$ & $\begin{array}{c}\text { \# BDD nodes of } \\
\text { transition function }\end{array}$ & $\begin{array}{c}\text { peak of \# BDD nodes of } \\
\text { Current/ Processed }\end{array}$ & $\begin{array}{c}\text { AWA } \\
\text { CPU time }\end{array}$ & $\begin{array}{c}\text { LIF } \\
\text { CPU time }\end{array}$ \\
\hline 2 & 21 & $10 / 11$ & $0.1 s$ & $0.0 s$ \\
4 & 59 & $34 / 42$ & $0.1 s$ & $0.0 s$ \\
8 & 189 & $318 / 717$ & $1.0 s$ & $4.0 s$ \\
10 & 269 & $971 / 3063$ & $8.2 s$ & $81.0 s$ \\
12 & 371 & $3438 / 13037$ & $71.7 s$ & $15241.5 s$
\end{tabular}

Table 1. Empirical results of the emptiness test for a $n$-bit counter

care must be taken in comparing these results: we have not included the time taken in constructing the AWA from the LIF system (it is linear) as this was done by hand. Further, Gupta and Fisher build a canonical representation of the LIF and they have used the older BDD package from David Long.

\section{Exponentially Inductive Boolean Functions}

The structure of this section parallels that of 93 . After defining EIFs, we show how their equality problem can be decided using tree automata. The decision procedure however is not as direct as it is for LIFs. One problem is that inputs to EIFs are words not trees. We solve this by labeling the interior nodes of trees with a dummy symbol. However, the main problem is that the words must be of length $2^{n}$. This restriction cannot be checked by tree automata and we solve this by deciding separately if a tree automaton accepts a complete tree.

\subsection{Definitions of EIFs}

Syntax An EIF formula (over $V$ and $F$ ) is a pair $(\alpha, \beta)$, with $\alpha \in B(V)$ and $\beta \in B(F \times\{L, R\})$. We write $f^{L}$ [respectively $f^{R}$ ] for the variable $(f, L)$ [respectively $(f, R)]$ in $F \times\{L, R\}$. An EIF system (over $V$ and $F$ ) is a pair $(\mathrm{F}, \eta)$, where $\mathrm{F}$ and $\eta$ are defined as for a LIF system. Similarly to LIF systems, we write $\left(\alpha_{f}, \beta_{f}\right)$ for $\eta(f)=(\alpha, \beta)$.

Semantics Let $\mathcal{S}$ be an EIF system over $V$ and $F$. An evaluation of $\mathcal{S}$ on a word $w=b_{1} \ldots b_{2^{n}} \in\left(\mathbb{B}^{r}\right)^{+}$is a complete binary $\mathbb{B}^{s}$-labeled tree $\tau$ with front $(\tau)=d_{1} \ldots d_{2^{n}} \in\left(\mathbb{B}^{s}\right)^{+}$and for $1 \leq k \leq s$ :

i) For $b_{i}=\left(a_{1}^{i}, \ldots, a_{r}^{i}\right)$ and $d_{i}=\left(c_{1}^{i}, \ldots, c_{s}^{i}\right)$, with $1 \leq i \leq 2^{n}$,

$$
c_{k}^{i}=1 \quad \text { iff } \quad\left\{v_{l} \mid a_{l}^{i}=1 \text {, for } 1 \leq l \leq r\right\} \models \alpha_{f_{k}} .
$$

ii) For each inner node $u \in \operatorname{dom}(\tau)$ with $\tau(u .0)=\left(c_{1}^{\prime}, \ldots, c_{s}^{\prime}\right), \tau(u .1)=$ $\left(c_{1}^{\prime \prime}, \ldots, c_{s}^{\prime \prime}\right)$, and $\tau(u)=\left(c_{1}, \ldots, c_{s}\right)$ :

$$
\begin{aligned}
c_{k}=1 \text { iff } \quad & \left\{f_{i}^{L} \mid c_{l}^{\prime}=1, \text { for } 1 \leq l \leq s\right\} \cup \\
& \left\{f_{i}^{R} \mid c_{l}^{\prime \prime}=1, \text { for } 1 \leq l \leq s\right\} \models \beta_{f_{k}} .
\end{aligned}
$$


Let $\Sigma^{2+}=\left\{w \in \Sigma^{*}|| w \mid=2^{n}\right.$, for some $\left.n \in \mathbb{N}\right\}$. As with LIFs, the evaluation $\tau$ is uniquely defined; hence $f_{k} \in F$ and $\mathcal{S}$ together define a function $f_{k}^{\mathcal{S}}:\left(\mathbb{B}^{r}\right)^{2+} \rightarrow \mathbb{B}$. Namely $f_{k}^{\mathcal{S}}(w)=c_{k}$, where $\tau$ is the unique evaluation of $\mathcal{S}$ on $w$ and $\tau(\lambda)=\left(c_{1}, \ldots, c_{s}\right)$. EIF-representable is defined analogously to LIFrepresentable.

For example, the tree implementation of the parameterized parity circuit from the introduction is described by the EIF system $\mathcal{S}$ over $V=\{x\}$ and

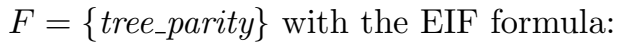

$$
\alpha_{\text {tree_parity }}=x \quad \beta_{\text {tree_parity }}=\text { tree_parity }^{L} \oplus \text { tree }_{-} \text {parity }{ }^{R} .
$$

Here the value of the EIF tree_parity ${ }^{\mathcal{S}}$ applied to a word $w=b_{1} \ldots b_{2^{n}} \in \mathbb{B}^{+}$is the value of the function parity $2^{n}$ applied to $\left(b_{1}, \ldots, b_{2^{n}}\right)$.

\subsection{Equivalence of EIF Systems and ATAs}

Using ATAs we can characterize the EIF-representable functions. To interpret a word in the domain of an EIF as a tree, we identify a word $b_{1} \ldots b_{2^{n}} \in \Sigma^{*}$ with the complete tree $t \in(\Sigma \uplus\{\#\})^{\mathrm{T} *}$, where front $(t)=b_{1} \ldots b_{2^{n}}$ and all inner nodes are labeled with the dummy symbol \#. In the following, $\Sigma_{\#}$ stands for $\Sigma \uplus\{\#\}$. We call a tree $t \in \Sigma_{\#}^{\mathrm{T} *}$ a $\Sigma$-leaf-labeled tree when (i) if $w$ is a leaf, then $t(w) \in \Sigma$ and (ii) if $w$ is an inner node, then $t(w)=\#$.

Normal forms for EIF systems can be defined and obtained as for LIF systems and the proof of Theorem [1] can, with minor modifications, be generalized to EIFs.

Theorem 3. EIF systems are equivalent to ATAs if the input trees are restricted to complete, leaf-labeled trees. In particular:

i) Let $\mathcal{A}=\left(\mathbb{B}_{\#}^{r}, Q, q_{0}, F, \delta\right)$ be an ATA. There is a normal form EIF system $\mathcal{S}$ over $V=\left\{v_{1}, \ldots, v_{r}\right\}$ and $Q$, such that for all $q \in Q$ and any complete $\mathbb{B}^{r}$-leaf-labeled tree $t \in\left(\mathbb{B}_{\#}^{r}\right)^{T+}$,

$$
q^{\mathcal{S}}(\text { front }(t))=1 \quad \text { iff } \quad \mathcal{A} \text { accepts } t \text { from } q .
$$

ii) Let $\mathcal{S}$ be a normal form EIF system over $V$ and $F$. There is an ATA $\mathcal{A}$ with the state set $F \uplus\left\{q_{\text {base }}, q_{\text {step }}\right\}$, such that $\mathcal{A}$ accepts from $f \in F$ only $\mathbb{B}^{r}$-leaf-labeled trees, and for any complete $\mathbb{B}^{r}$-leaf-labeled tree $t \in\left(\mathbb{B}_{\#}^{r}\right)^{T+}$,

$$
\mathcal{A} \text { accepts } t \text { from } f \quad \text { iff } \quad f^{\mathcal{S}}(\text { front }(t))=1 \text {. }
$$

\subsection{Deciding EIF Equality}

The equality problem for EIFs and the size of an instance are defined similarly to LIFs. We cannot generalize the decision procedure from \$3.3 to EIFs since we are only interested in trees of a restricted form: complete leaf-labeled binary trees. Unfortunately completeness is not a regular property, i.e. one recognizable 
by tree automata, and hence we cannot reduce the problem to an emptiness problem. Instead, we reduce the problem to the complete-tree-containment problem (CTCP) for NTAs, which is to decide whether a given NTA accepts a complete tree.

Theorem 4. The equality problem for EIFs is in EXPSPACE.

Proof. Let $\mathcal{S}$ over $V$ and $F$, and $\mathcal{T}$ over $V$ and $G$ be EIF systems, and let $f \in F$ and $g \in G$ be given. Let $\widetilde{\mathcal{S}}$ be the EIF system over $V$ and $\{\widetilde{f}\} \uplus F \uplus G$ with the additional EIF formula $\alpha_{\tilde{f}}=\neg\left(\alpha_{f} \leftrightarrow \alpha_{g}\right)$ and $\beta_{\widetilde{f}}=\neg\left(\beta_{f} \leftrightarrow \beta_{g}\right)$. We normalize $\widetilde{S}$, and by Theorem 3 (ii) construct an ATA $\mathcal{A}$ with the initial state $\tilde{f}$, such that $f^{\mathcal{S}} \neq g^{\mathcal{T}}$ iff $\mathcal{A}$ accepts a complete tree. $\mathcal{A}$ has $2|\{\tilde{f}\} \uplus F \uplus G|+2$ states and the size of the alphabet $\mathbb{B}_{\#}^{|V|}$ is $2^{|V|}+1$. From $\mathcal{A}$ we can construct an equivalent NTA $\mathcal{B}$ that has at most $O\left(2^{2|F|+|G|}\right)$ many states. Hence we have reduced the equality problem for EIFs to CTCP for NTAs. The required space for the reduction is $O\left(2^{|V|} 2^{2|F|+|G|}\right)$.

We now show that CTCP for NTAs is in PSPACE. For the NTA $\mathcal{A}=$ $\left(\Sigma, Q, q_{0}, F, \delta\right)$ we construct the AWA $\mathcal{A}^{\prime}=\left(\{1\}, Q, q_{0}, F, \delta^{\prime}\right)$ with $\delta^{\prime}(q, 1)=$ $\bigvee_{a \in \Sigma} \bigvee_{\left(p, p^{\prime}\right) \in \delta(q, a)}\left(p \wedge p^{\prime}\right)$. It is easy to prove that $\mathcal{A}$ accepts a complete tree of height $h$ iff $\mathcal{A}^{\prime}$ accepts a word of length $h$. From this follows that CTCP for NTAs is in PSPACE because the emptiness problem for AWAs is in PSPACE [12 17]. $\square$

In [13, it is proved that the equality problem for EIFs and CTCP for ATAs (to decide if an ATA accepts a complete tree) are both EXPSPACE-hard. We omit the proof, which is quite technical, due to space limitations.

\section{Comparisons and Related Work}

Our work was motivated by that of Gupta and Fisher [6/7/8] and we begin by comparing our LIFs and EIFs with theirs, which we will call $\mathrm{LIF}_{0}$ and $\mathrm{EIF}_{0}$.

For each $n \geq 1$, a $\operatorname{LIF}_{0} f$ is given by a Boolean function, called the $n$-instance of $f$ and denoted by $f^{n}$, where $f^{1}: \mathbb{B}^{r} \rightarrow \mathbb{B}$ and $f^{n}: \mathbb{B}^{r+s} \rightarrow \mathbb{B}$ for $n>1(r$ is the number of $n$-instance inputs and $s$ is the number of $(n-1)$-instance function inputs). Further it must hold that for all $m, n>1$ the $m$-instance and the $n$ instance of $f$ are equal, i.e. $f^{m}=f^{n}$. By means of the parity function we explain how the value of a $\mathrm{LIF}_{0}$ is calculated. The $n$-instances of serial_parity ${ }^{n}$ (using their notation) are:

$$
\begin{aligned}
& \text { serial_parity }^{1}=b^{1} \text {, } \\
& \text { serial_parity }^{n}=b^{n} \oplus \text { serial_parity }^{n-1} \quad \text { for } n>1 \text {. }
\end{aligned}
$$

The value of the $\mathrm{LIF}_{0}$ serial_parity on the word $b_{1} \ldots b_{n} \in\left(\mathbb{B}^{r}\right)^{+}$, written as serial_parity $\left(b_{1}, \ldots, b_{n}\right)$, is the value of the 1-instance serial_parity ${ }^{1}$ applied to $b_{1}$ for $n=1$. For $n>1$, it is the value of the $n$-instance serial_parity ${ }^{n}$ applied to $b_{n}$ and serial_parity $\left(b_{1}, \ldots, b_{n-1}\right)$. 
The definitions of a "LIF formula" and a "LIF system" correspond to the definition of a "LIF 0 ". Moreover, the way the "value" of a $\mathrm{LIF}_{0}$ is calculated corresponds to our definition of "evaluation". Hence both formalisms are equivalent. However, the algorithms, data-structures, and complexity of our approaches are completely different!

Gupta and Fisher formalize $\mathrm{LIF}_{0}$ s using a data-structure based on BDDs where terminal nodes are not just the constants 0 and 1 , but also pointers to other BDDs. They then prove that each LIF system has a canonical representation that can be obtained in $O\left(2^{2^{|F|}} 2^{|V|}+\left(2^{2^{|F|}}\right)^{2}\right)$ time and space in the worst-case. In contrast, we have given a decision procedure (Theorem 2) that requires polynomial space, which is a doubly exponential improvement in space and an exponential improvement in time. Despite its worse space complexity, our algorithm based on BDD-represented AWAs may give better results in practice than our PSPACE decision procedure. This depends on whether the BDDs used require polynomial or exponential space. If the space required is polynomial, then the resulting AWA and its emptiness test require only polynomial space. In the exponential case, as there are only $2|F|+2$ states, the emptiness test requires $O\left(2^{|V|+2|F|}\right)$ time and space. This case also represents an exponential improvement over Gupta and Fisher's results, both in time and space.

An $\mathrm{EIF}_{0} f$ has, like a $\mathrm{LIF}_{0}$, for each $n \geq 0$, a $n$-instance function $f^{2^{n}}$, where $f^{1}: \mathbb{B}^{r} \rightarrow \mathbb{B}$ and, for $n>0, f^{2^{n}}$ is a Boolean combination of three $\mathrm{EIF}_{0} \mathrm{~s}, e, g$ and $h$, i.e. $f^{2^{n}}: \mathbb{B}^{3} \rightarrow \mathbb{B}$. Further it must hold that $f^{2^{m}}=f^{2^{n}}$ for all $m, n>0$. The value of the $\operatorname{EIF}_{0} f$ on the word $b_{1} \ldots b_{2^{n}} \in\left(\mathbb{B}^{r}\right)^{+}$, written as $f\left(b_{1}, \ldots, b_{2^{n}}\right)$, is the value of the 0 -instance $f^{1}$ applied to $b_{1}$ if $n=0$. For $n>0$, it is the value of the $n$-instance $f^{2^{n}}$ applied to the value of the $\operatorname{EIF}_{0} e$ of the left half of the word, i.e. $e\left(b_{1}, \ldots, b_{2^{n-1}}\right)$, and to the values of the $\mathrm{EIF}_{0} \mathrm{~s} g$ and $h$ of the right half of the word, i.e. $g\left(b_{2^{n-1}+1}, \ldots, b_{2^{n}}\right)$ and $h\left(b_{2^{n-1}+1}, \ldots, b_{2^{n}}\right)$.

$\mathrm{EIF}_{0} \mathrm{~s}$ are strictly less expressive than EIFs. Indeed, since not every Boolean function for the $n$-instance function of an $\operatorname{EIF}_{0}$ (for $n>0$ ) is allowed, even simple functions cannot be described by an $\mathrm{EIF}_{0}$, e.g., $F: \mathbb{B}^{2+} \rightarrow \mathbb{B}$ with $F(w)=1$ iff $w=0000$ or $w=1100$ or $w=1011$. The reason is similar to why deterministic top-down tree automata are weaker than nondeterministic top-down tree automata; the restrictions of the $n$-instance function of an $\mathrm{EIF}_{0}$ stems from the data-structure proposed for $\mathrm{EIF}_{0} \mathrm{~s}$ in [6] in order to have a canonical representation. On the other hand, it is easy to see that $F$ is EIFrepresentable.

Our results on the complexity of the equality problem for EIFs are, to our knowledge, the first such results given in the literature. Neither we nor Gupta and Fisher have implemented a decision procedure for the equality problem for EIFs or $\mathrm{EIF}_{0} \mathrm{~s}$.

We have seen that LIFs and EIFs can be reduced to word and tree automata. Gupta and Fisher also give in [6] an extension of their data-structure for $\mathrm{LIF}_{0} \mathrm{~S}$ and $\mathrm{EIF}_{0} \mathrm{~s}$ that handles more than one induction parameter with the restriction that the induction parameters must be mutually independent. We conjecture that it may be possible to develop similar models in our setting based on 2- 
dimensional word automata (as described in [5]) and their extension to trees. However, this remains as future work.

There are also similarities between our work and the description of circuits by equations of Brzozowski and Leiss in [2]. A system of equations $S$ has the form $X_{i}=\bigcup_{a \in \Sigma}\{a\} . F_{i, a} \cup \delta_{i}$ (for $1 \leq i \leq n$ ) where the $F_{i, a}$ are Boolean functions in the variables $X_{i}$, and each $\delta_{i}$ is either $\{\lambda\}$ or $\emptyset$. It is shown in [2] that a solution to $S$ is unique and regular, i.e., if each $X_{i}$ is interpreted with $L_{i} \subseteq \Sigma^{*}$ and the $L_{i}$ satisfy the equations in $S$, then the $L_{i}$ are unique and regular. LIF systems offer advantages in describing parameterized circuits. For example, with LIFs one directly describes the "input ports" using the variables $V$. In contrast, a system of equations must use the alphabet $\mathbb{B}^{r}$ and cannot "mix" input pins and the signals of the internal wiring (and the same holds for outputs). Furthermore, descriptions using LIFs cleanly separate the base and step cases of the circuit family, which is not the case with 2 .

Finally, note that the use of BDDs to represent word and tree automata, without alternation, is explored in 10,14. There, BDD-represented automata are used to provide a decision procedures for monadic second-order logics on words and trees. This decision procedure is implemented in the MonA system, and MonA can be used to reason about LIF systems [1]: a LIF system is described by a monadic second-order formula, which MonA translates into a deterministic word automaton. Although this has the advantage of using an existing decision procedure, the complexity can be considerably worse both in theory and in practice. For example, for a 12-bit counter MoNA (version 1.3) needs more than an hour to build the automaton and the number of BDD nodes is an order of magnitude larger than what is needed for our emptiness test for AWAs.

\section{Conclusions}

We have shown that LIFs and EIFs can be understood and analyzed using standard formalisms and results from automata theory. Not only is this conceptually attractive, but we also obtain better results for the decision problem for LIFs and the first complexity results for EIFs. The $n$-bit counter example in $\$ 3.3$ indicates that our approach, at least in some cases, is faster in practice. However, an in depth experimental comparison of the procedures remains as future work.

Acknowledgments: The authors thank Aarti Gupta for helpful discussions and kindly providing us with recent timings using her package. We also thank the referees for their suggested improvements.

\section{References}

1. D. Basin and N. Klarlund. Automata based symbolic reasoning in hardware verification. The Journal of Formal Methods in Systems Design, 13(3):255-288, 1998.

2. J. Brzozowski and E. Leiss. On equations for regular languages, finite automata, and sequential networks. TCS, 10(1):19-35, 1980. 
3. A. Chandra, D. Kozen, and L. Stockmeyer. Alternation. Journal of the ACM, 28(1):114-133, 1981.

4. F. Gécseg and M. Steinby. Tree Automata. Akadémiai Kiadó, Budapest, 1984.

5. D. Giammarresi and A. Restivo. Two-dimensional languages. In A. Salomaa and G. Rozenberg, editors, Handbook of Formal Languages, volume 3, Beyond Words, chapter 4, pages 215-267. Springer-Verlag, 1997.

6. A. Gupta. Inductive Boolean Function Manipulation: A Hardware Verification Methodology for Automatic Induction. PhD thesis, School of Computer Science, Carnegie Mellon University, Pittsburgh, 1994.

7. A. Gupta and A. Fisher. Parametric circuit representation using inductive boolean functions. In $C A V$ 93, volume 697 of $L N C S$, pages 15-28, 1993.

8. A. Gupta and A. Fisher. Representation and symbolic manipulation of linearly inductive boolean functions. In Proc. of the IEEE Internation Conference on Computer-Aided Design, pages 192-199. IEEE Computer Society, 1993.

9. A. Gupta and A. Fisher. Tradeoffs in canonical sequential representations. In Proc. of the International Conference on Computer Design, pages 111-116, 1994.

10. J. Henriksen, J. Jensen, M. Jorgensen, N. Klarlund, B. Paige, T. Rauhe, and A. Sandholm. Mona: Monadic second-order logic in practice. In TACS 95, volume 1019 of $L N C S$, pages 89-110, 1996.

11. J. Hopcroft and J. Ullman. Formal Languages and their Relation to Automata. Addison-Wesley, 1969.

12. T. Jiang and B. Ravikumar. A note on the space complexity of some decision problems for finite automata. IPL, 40(1):25-31, 1991.

13. F. Klaedtke. Induktive boolesche Funktionen, endliche Automaten und monadische Logik zweiter Stufe. Master's thesis, Institut für Informatik, Albert-LudwigsUniversität, Freiburg i. Br., 2000. in German.

14. N. Klarlund. Mona \& Fido: The logic-automaton connection in practice. In $C S L$ 97, volume 1414 of $L N C S$, pages 311-326, 1998.

15. G. Slutzki. Alternating tree automata. TCS, 41(2-3):305-318, 1985.

16. F. Somenzi. CUDD: CU Decision Diagram Package, Release 2.3.0. Department of Electrical and Computer Engineering, University of Colorado at Boulder, 1998.

17. M. Vardi. An automata-theoretic approach to linear temporal logic. In Logics for Concurrency, volume 1043 of LNCS, pages 238-266, 1996. 